\title{
Medical training in the UK
}

\section{Stewart}

\section{Time for a change?}

T his paper describes the potential consequences of the reduced duration of the training grades in combination with the reduction in working hours and centralisation of paediatric intensive care, on the level of expertise at appointment to consultant as paediatricians in dealing with critically ill children.

The lack of change in educational method has failed to compensate for these changes with no lowering of public expectation. This is an unfair position in which to place doctors in and represents an unacceptable risk to patients. A potential solution is proposed involving a restructuring of trainee rotations, the inclusion of Paediatric Intensive Care Medicine into the General Paediatric Requirement for Higher Specialist Training as is Neonatal Intensive Care, the establishment of hi fidelity simulation centres in all lead centres, and lo fidelity simulation centres at all hospitals to form an educational matrix increasing the efficiency of training and allowing for an integrated approach to critical illness previously unobtainable.

Medicine continues to increase in complexity, depth, breadth, and level of interventions possible. The speed of this change in medicine and society has overtaken our current system of development in education and training. This has

\section{Key points}

- Calman and the reduction in junior doctors hours have significantly reduced the time available for training

- Efficiency of training has not kept pace

- The move to a consultant based service further reduces the ability of consultants to fulfil trainee educational requirements

- Regionalisation of paediatric critical care reduces exposure to incipient and actual critical illness outside of lead centres

- Mandatory rotations through PICU can offset the lack of exposure

- Hi fidelity simulation centres can be used to increase the efficiency of training, compensate for the reduced time allowed for training, maintain skills, and guarantee exposure to a core curriculum of critical illness lead to an increasing gap between expectation and the ability to deliver.

While centred on Paediatric Intensive Care Medicine, and the critically ill child, the theme suggested is transferable to other disciplines.

\section{THE CHANGES IN MEDICAL EDUCATION}

Postgraduate medical training in the UK has undergone radical changes in the past 10 years. The actual length of time allowed for training has been reduced by approximately half. During this time the required knowledge base has increased significantly. Despite dedicated consultants, techniques of postgraduate medical education have not kept pace with these changes, while public expectation, litigation, and governmental pressure continue to expect perfection.

Teaching is an inherent part of medicine. There is still heavy reliance on one to one teaching at the bedside, supplemented by lectures and tutorials to guide the trainee in their self development. The drive and ambition of the trainee remains central to their acquisition of much of the background knowledge base.

The organisation of rotations has been improved, enabling the acquisition of relevant experience while reducing redundancy of placement. However, despite the hard work of consultants and postgraduate deans, the educational system and training received within them has not developed as fast. Teaching methodology has remained essentially unchanged and training the trainers is still in its infancy. Technology has crept into some areas but it generally remains an accessory.

The result is that little improvement has been made in increasing the efficiency of education despite less time in which to teach. This is compounded by the continued drift towards a consultant based delivery of care rather than a consultant led system, leaving even less time for education. Adding the consultants' own requirement for continuing professional development, which has been estimated to take 1.5 sessions per week to administer, the time available for trainees is vanishing fast. This is most keenly felt in the acute specialties. In these, the introduction of shift systems precludes a traditional lecture/tutorial approach, as rarely are there more than a fraction of the trainees available at any one time.
We are in danger of a reduction in training time approaching that of the North American system, where, after higher specialist training comprising a three year Fellowship, one can look for specialist jobs. However, while the knowledge base of these individuals is high because of the structured methodology, their experience level (relative to the British graduate) is low.

The result is handled differently in different States, and is not usually formalised, but essentially produces the "junior attending" (junior consultant) grade, occasionally with a limitation on "privileges" (what you can and cannot do), which allows the development of sufficient experience prior to "senior" consultant status.

While not advocating the North American system, they have at least recognised that in order to be a successful (and safe) practitioner, you require both knowledge and experience, having structured their training programmes and career paths accordingly.

The change in our training programme with the introduction of Calman compounded by the European Working Hours Directive has failed to maintain this balance. With greater emphasis being placed on demonstrating quality of care and the introduction of Clinical Governance, levels of competence for any practice in medicine today are being defined. In keeping with this, we are moving from a time based apprenticeship to a competence based apprenticeship. Yet our current system does not allow for efficient education of hospital based practitioners. There are two main components to a potential solution for paediatrics. Firstly, the rotations undertaken, secondly the methods used to educate and train.

\section{WHY CHANGE ROTATIONS?}

The regionalisation of critical care services, supported by a retrieval system, while a necessary step for efficient service delivery, has the knock on effect of reducing further the exposure of trainees to critically ill patients for resuscitation and stabilisation outside the lead centres. The general paediatrician remains core to the resuscitation and stabilisation of critically ill children. Unless a period of time is also spent in the paediatric intensive care unit (PICU), the experience of recognition and prevention of physiological decompensation has been reduced. Just as neonatal intensive care has become a discipline both independent yet intrinsic to general paediatric education, including a concentrated attachment in Paediatric Intensive Care Medicine has now become a necessity.

However, a guarantee of clinical exposure to critically ill (paediatric) patients could be seen to be an essential part of 
higher specialist training for a number of disciplines. General paediatrics, paediatric $A \& E$, and paediatric anaesthesia are obvious, but there are others who would benefit from critical care training in order to improve morbidity by preventing decompensation of sick children. The paediatric surgical trainee, paediatric cardiologist, and adult anaesthetist who works in a district general hospital (DGH) would all have a better experience base to draw on in crisis management and, as importantly, its prevention.

As such, the last or penultimate part of rotations for which there is a need could be via the departments of paediatric intensive care medicine. Duration of training is obviously debatable. The Intercollegiate Committee on Paediatric Intensive Care Medicine (ICTPICM) has correctly left aside the requirements for non-intensivists, and has begun to accredit training centres from the viewpoint of training dedicated paediatric intensivists. All lead centres for paediatric intensive care, however, rely on non-intensivist trainees to service the units. The dedicated intensivist trainee forms a tiny minority of the trainees in any unit at any one time. Thus the education and training requirements of the majority of trainees in these units are not being specifically addressed. It could be argued that the general paediatrician who may be responsible for the resuscitation and stabilisation of critically ill children should have no less than six months, and preferably 12 months exposure. Likewise the paediatric anaesthetist or adult anaesthetist who intends to work with children.

While the inclusion of the PICU within rotations will provide exposure to critical illness, it would be naïve to believe that the duration of that exposure will be sufficient given current training techniques. Thus the methodology of training must also change.

Until now, exposure to specific experiences, from crisis management (advanced airway control, cardiopulmonary resuscitation, etc) to the management of conditions such as meningococcal disease, head injury, viral myocarditis, multiple trauma, etc has been down to luck and duration of the attachment. Even intubation skills can be difficult to gain exposure to, when in competition with trainee anaesthetists, as the number of available opportunities is limited. The simulation centre offers a way of guaranteeing this experience.

This guaranteed exposure is combined with educational modalities designed to increase the efficiency of learning, to make best use of the limited time available. This utilises interactive teaching methods, based on multimedia computers, and small group interactions centred on problem based learning of scenarios with rapid positive feedback of performance. A rotation through a simulation centre early in a person's career, at SHO level perhaps, could help provide a more coherent base for crisis management in their registrar days to come.

\section{WHY SIMULATORS?}

The majority of clinicians base their assessment of patients on two elements: the clinical history, and the "gestalt" summation of the clinical appearance, examination, and history together. This is more likely the more complex the case, because of the large number of parameters requiring integration in order to arrive at the correct clinical decision. It is easier to form a memory of many complex parts by hanging them all on a single framework, the patient. Thus bedside teaching is a vital and irreplaceable component of medical education. It also requires a suitable patient on which to hang the clinical picture. With a decreasing number of patients available per trainee at junior level, faster throughput, and reduced time as a trainee, this is endangered.

Furthermore, the increased number and complexity of interventions now expected places the trainee in the uncomfortable position of handling them without sufficient practical experience. In today's climate of litigation, media harassment, and high public expectation, this is unacceptable. Thus a simulated patient, which is close enough to reality to allow for suspension of disbelief without so much effort that it detracts from the learning experience, is an effective way of increasing clinical exposure to a greater variety of problems with greater reliability.

One must remain cognisant of the fact that in the majority of hospitals, resuscitation and stabilisation of critically ill children is undertaken by general paediatricians, usually with anaesthetic trainee and consultant assistance. With concerns over surgical safety, anaesthetists are seeing fewer children as routine outside specialist centres. Not only is this reduction in clinical exposure at trainee level worrying, but also some consultant anaesthetists in district general hospitals are becoming more uncomfortable in intubating seriously ill children when they do present. The scenario of a consultant being appointed in the next few years as a paediatrician in a DGH who may be called to the accident and emergency department to resuscitate or stabilise a critically ill child with only the APLS course they took three years previously as experience, is an uncomfortable one. The trainees will be looking to that person for leadership, guidance, and possibly interventional skills as well. In today's society, this is neither an acceptable position to place a new consultant in, nor an acceptable risk for the patient.

The human patient simulator, while still imperfect, is a significant improvement over anything previously available.
It is, however, not the sole component required to improve training. It is in the setting of a clinical simulation centre, which pulls together all the elements of education and training into a package that can be efficiently delivered, monitored, and refined to maximise the trainees' learning experience. The airline industry has recognised this for years and it would be unthinkable that a pilot would take to the controls of a new aircraft without spending time in a simulator first. Public expectations continue to rise, and the consequences of failing to meet these expectations are become ever more draconian. The simulation centre offers a means to improve the maintenance of skills, learn practical procedures in a safe environment, enhance teamwork, and offer a method of evaluating and assessing the ability of clinicians to deal with complex situations which are closer to that clinician's real life work than ever before. It has a significant role to play in dealing with human factor issues and error control. The simulation centre offers us a means to enhance the veracity of our internal quality control, rather than have one imposed on us from outside. The simulator must, however, be complimented by procedural trainers, knowledge base trainers, and assessment and evaluation tools of that knowledge base. Appendix 1 contains a brief overview of the components of a simulation centre.

\section{CONCLUSIONS}

If the present situation continues, we are facing an ever widening gap between public and governmental demand for perfection on the one hand and reality on the other.

The introduction of widespread use of the simulation centre may improve the efficiency of training to allow for an increase in competence and maintenance of skills, which will improve patient care. As a spin off, a reduction in individual stress and improvement in team functioning may improve morale. Integration of rotations to allow intensive care medicine to form a required part of training will also mean that the sickest of patients will have a more integrated, seamless quality of care from presentation at the accident and emergency department to discharge, whether that was via a transfer to PICU or not. General practitioners can benefit from this system as well in the maintenance of crisis management skills, adding to the overall integration of the delivery of health care to the population.

This solution, while superficially costly, requiring central funding, is a direct consequence of the direction society has lead medicine to take. The RCPCH could take a lead by making Paediatric Intensive Care Medicine a mandatory part of training, just as Neonatal 
Intensive Care Medicine is. In reality it would be hoped that the costs could be offset by the reduction in litigation, improvements in competence, and the ability to assess competence throughout training in a more appropriate way than previously available.

Appendix 2 summarises the educational issues raised by this paper and one possible way of developing this concept into an integrated National Strategy to improve quality of education, training, and thus patient care. The financial implications are not addressed.

\section{APPENDIX 1 \\ Simulation centre educational components}

(This does not include the personnel, management, and support services the centre requires to function.)

- Procedural trainers (intubation, vascular access, thoracostomy, etc)

- Interactive knowledge base computer systems

- Lo fidelity simulator for crisis management education and maintenance of skills

- Hi fidelity simulator for complex pathophysiology training, and assessment and evaluation of performance

- Research and development laboratory.

Procedural training suite

While the simulator, comprising the mannequin and computer are at the core, they are part of an integrated package. The procedural training suite contains skill stations with specialised, relatively inexpensive, mannequins to learn practical procedures, from intubation to central line placement, and practice crisis interventions on (once the knowledge base for the procedure has been gained).

\section{Knowledge base trainers}

The use of the simulator obviously requires a suitable knowledge base to be an effective learning experience. This requires a computer suite for computer based interactive tutorials and educational programmes. These are developing to include automated reporting of performance to a remote server, allowing the trainer to monitor and assess the individual's developing knowledge base. These systems also free the trainee from the time constraints of the traditional timetable, being able to utilise their "quiet time" with these teaching programmes. As more and more use is made of the internet and distance learning, connections for "e-learning" will also help increase an individual teacher's ability to reach a larger audience. Conversely, individual trainees can access training materials and highly experienced teachers at their convenience. A seminar room is still required for tutorials to deal with questions not answered by the automated tutorials, and a lecture theatre is still needed for larger group work and demonstrations preparing the trainees for the use of the simulator.

\section{The simulator system}

Both the hi and lo fidelity simulators share the same basic operational systems. They have at their core a computer containing mathematical models of human physiology. These output through a mannequin. The mannequin is capable of simulating human physiological responses to a variety of situations. "Normal" behaviour such as breathing, consuming oxygen, producing carbon dioxide, pulses, heart sounds, and pupillary responses are all present and change in response to the different clinical scenarios programmed in. Thus if you listen to the chest you hear the breath sounds, if there is a "tension pneumothorax", they will change as they do in a real patient. If you talk to the patient, it can also talk and tell you of their difficulty in breathing. This will also manifest itself in the respiratory rate, cardiovascular changes, and blood gas picture and, if no intervention is made, the resulting cardiorespiratory arrest.

Drug effects are also simulated, and any drug can be programmed in once the pharmacokinetics are known. Indeed these simulators are used to train anaesthetists in the use of new pharmacological agents, including inhalational anaesthetics.

The physical capabilities are also much improved over the mannequins of old. The difficult airway can include airway obstruction from oedema and laryngospasm. Trauma victims can be simulated, and the military have made much use of these. Other procedures such as needle thoracocentesis, chest drain placement, cricothyrotomy, pericardiocentesis, etc can be performed. The company METI manufactures these systems. They come in adult and paediatric versions. There is also a simulator produced by Laerdal (Sim-man, comparable to the METI lo fidelity simulator). This one differs in that each response the mannequin produces has to be programmed into a "script" which the mannequin follows. This is adequate for simple crisis management, but much less suited to complex pathophysiology than the automatic responses of the mathematical model driven system; a paediatric version of the Sim-man is awaited.

The lo fidelity simulator

The hi fidelity simulator is necessary for complex pathophysiological problems. However, simple crisis management does not require the same level of sophistication. Thus a lo fidelity simulator, which lacks some of the more sophisticated abilities such as pericardiocentesis, but is significantly cheaper, will suffice. Crisis management along the lines of the APLS/PALS scenarios is further enhanced by the use of the lo fidelity simulator. Use of the lo fidelity simulator can be integrated into the role of the resuscitation training officer.

The hi fidelity simulator

The hi fidelity simulator is well suited to the simulation of complex pathophysiological scenarios. It can therefore be used in teaching the management of complex critical illness. It can also be used in the assessment and evaluation of performance, as well as education of basic physiology and pathophysiology. It therefore can be used in the education and maintenance of skills in medical students, trainee grades, advanced nurse practitioners, and consultants.

\section{The learning environment}

The simulator is usually placed in an authentic environment, a recreation of an operating room, intensive care ward, or accident and emergency room. All the simulated scenarios are recorded in detail: all the physiological changes and interventions, together with video and audio of the actual performance of the trainees. This is debriefed in adjacent rooms linked by full AV connections allowing the replay of the scenario to facilitate an effective and positive learning experience. Observance of simulated scenarios also plays a part in the preparation. A research laboratory is a necessary part of the system to allow for the continued refinement and development of the training packages provided, as well as to undertake research afforded by the simulation algorithms. The simulator's forte is in the presentation of complex problems in a controlled environment to both educate and evaluate performance. They are not perfect, but are steadily improving. The concept, however, appears sound.

\section{Infrastructure}

In order for the system to perform, the infrastructure must be in place to support its use. This means the employment of a manager, secretarial support, dedicated technician, and director. There must be funds available for the time of other clinicians, mainly consultants, but also SpRs and clinical nurse specialists to run scenarios.

\section{APPENDIX 2}

Educational scheme (for paediatrics) Any solution to the problems outlined in this paper must address the following issues.

- Maintenance of the level of knowledge and experience gained during the shortened hours of work available to trainees.

- Maintenance of the high level of skill and ability in new consultants with shorter training schemes. 
- Maintenance of the appropriate level of ability in: resuscitation, crisis management, and the recognition of impending decompensation for physicians (especially in DGHs, where an individual's exposure to crisis events is infrequent).

- Improvement in, and maintenance of crisis management skills in all health care providers in all wards in the face of staff shortages and infrequency of events.

- Improvement in the provision and integration of care to the critically ill paediatric patient irrespective of their location.

One possible schema to achieve this is outlined below. While constructed as an integrated whole, it could be implemented in a staged manner. This is of course a significant and contentious issue with many possible solutions, all of which will undoubtedly require substantial funding, at least in the short term.

(1) Introduction of hi fidelity simulation centres for each lead centre of paediatric intensive care.

(2) Introduction of lo fidelity simulation centres for every hospital receiving inpatients.

(3) Short attachment at SHO level for crisis management training (lo fidelity). This should be multidisciplinary and include the nursing staff

(4) Mandatory rotations through the hi fidelity simulation centre for $1-2$ months for all SpRs, early on (year 1-2). They would be involved in running more complex pathophysiological scenarios, crisis management, and would be expected to write a new scenario. (Does not preclude participation in an on call rota with following day off, giving four days per week in the simulator.)

(5) Mandatory rotations through the PICU for all appropriate (nonintensivist) SpRs towards the end of their training (6-12 month attachment).

(6) Development of a set of competences for crisis management in each subspecialty.

(7) Development of knowledge base assessment and evaluation tool in order to monitor the performance of the system as well as deliver an objective report to the trainee of their performance. These would be undertaken on admission and exit of an attachment to the system. This could be modular in nature to allow flexibility to reflect the expectations of each form of attachment.

(8) Mandatory rotation for all medical staff who have patient contact through the lo fidelity simulator for maintenance of crisis management skills. Capacity of each centre to allow for each person to have a one hour maintenance session every 3-4 months. These should be multidisciplinary, team based scenarios. The resuscitation training officer would be central to this undertaking.

This can form the basis for an expansion of the integration of critically ill patient care in the following way.

(1) Link lead centres with their dependent regional district hospitals with telemedicine links (mobile base station at dependent hospital)

(2) Provide all hospitals with lo fidelity simulators for crisis management training and maintenance of skills, including AV facility for debrief.

(3) Utilise the telemedicine links to allow the lead centre to facilitate scenarios on the DGH's lo fidelity simulator, and the DGHs to facilitate scenarios on the lead centre's hi fidelity simulator. (The simulator AV data should be transmissible directly from the AV system itself.) This should allow for greater integration of resuscitation, stabilisation, and transfer procedures.

(4) Extend the system to include paramedics, who can carry remote telemedicine links (wireless helmet mounted camera and microphones are already available), allowing the base receiving hospital doctors to engage in the assessment and treatment of the patients at the scene (which may be invaluable with trauma or near miss cot deaths, for example), by remote. This may allow for greater preparation to receive the patient and also allow the paramedics more appropriate advice if required.

\section{Arch Dis Child 2003;88:655-658}

Correspondence to: Dr D Stewart, Lead Clinician for Training \& Clinical Development, Manchester Children's University Hospitals, Hospital Road, Pendlebury, Manchester M274HA, UK; dstewart@chla.usc.edu Department of Intensive Care Medicine

\section{REFERENCES}

1 Lester H. Tritter JQ. Medical error: a discussion of the medical construction of error and suggestions for reforms of medical education to decrease error. Med Educ 2001;35:855-61.

2 Mclndoe A. Clinical feature article. The future face of medical training ship-shape and Bristol fashion. NATNEWS Nov 1988.

3 Byrnes AJ, Hilton PJ, Lunn JN. "ACCESS" - anaesthetic computer controlled emergency situation simulator. Basic simulations for anaesthetists-a pilot study of the ACCESS system. Anaesthesia 1994:49:376-81.

4 Department of Health. The new NHS modern and dependable: a national framework for assessing performance. Consultation paper, 1998

5 Green Paper. Our healthier nation. A contract for health. London: The Stationery Office, 1998 (ISBN-010138522 6).

6 Howard SK, Gaba DM, Fish KJ, et al. Anaesthesia crisis resource management training: teaching anaesthesiologists to handle critical incidents. Aviat Space Environ Med 1992;63:763-70.

7 Schwid HA, Rooke GA, Ross BK, et al. Use of a computerised ACLS simulator improves performance of ACLS guidelines. Anaesthesiology 1997;87:A934

8 Lurie S. Innovation and service traditional at University of Michigan Medical School. JAMA 2000;283:865-6.

9 Tome JA, Fletcher J. Virtual PBL: full-scale human simulation technology. Acad Med 1996;71:523.

10 Byrick R, Cleave-Hogg D. A crisis management program for residents in anesthesia. Acad Med 1998;73:592.

11 Patel RM, Crobleholme WR. Using simulation to train residents in managing critical events. Acad Med 1998;73:593.

12 Elliott S, Gordon JA. Integration of self-directed computerised patient simulations into the internal medicine ambulatory clerkship. Acad Med 1998:73:611.

13 Cavanaugh S. Computerised simulation technology for clinical teaching and testing. Acad Med 1997:4:939-43.

14 McGee JB, Neill J, Goldman L, et al. Using multimedia virtual patients to enhance the clinical curriculum for medical students. In Cesnick $B$, et al, eds. MEDINFO 98, 732-5.

15 Wyatt JP. Weber JE. A transatlantic comparison of training in emergency medicine. Emerg Med J 1998;15:175-80.

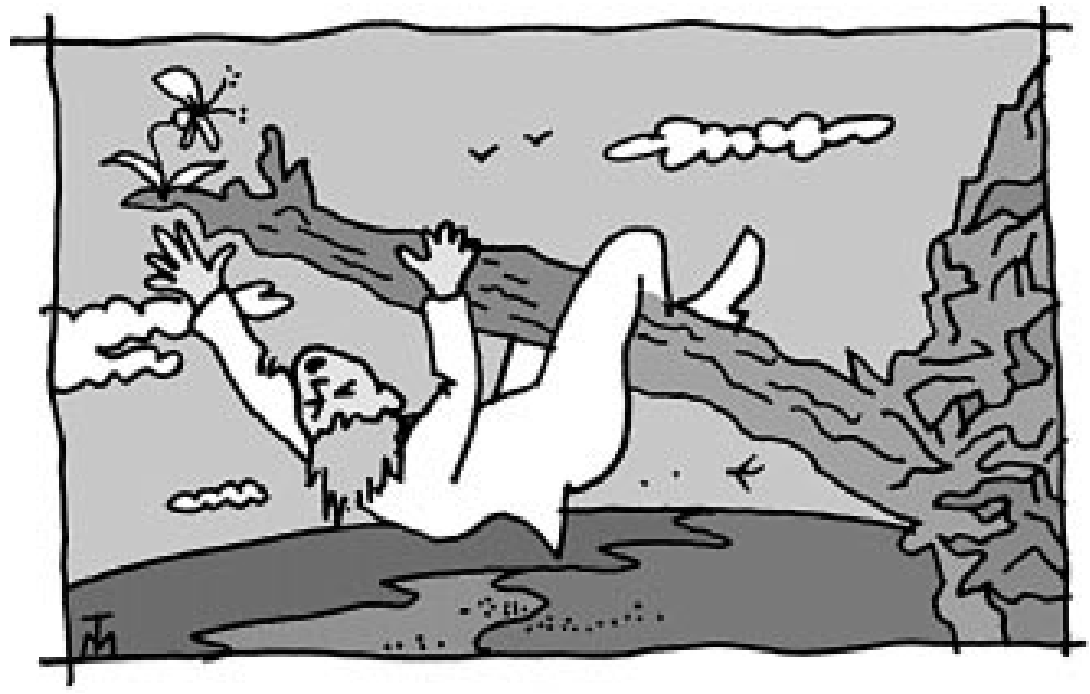

Illustration by Terry McElroy 